\title{
Severity Two Out of Five
}

National Cancer Institute

\section{Source}

National Cancer Institute. Severity Two Out of Five. NCI Thesaurus. Code C147504.

A severity result of two, on a five level scale. 